\title{
Ceres internal structure from geophysical constraints
}

\author{
Scott D. KING (D) ${ }^{1, *}$, Julie C. CASTILLO-ROGEZ (D) ${ }^{2}$, M. J. TOPLIS ${ }^{3}$, Michael T. BLAND ${ }^{4}$, \\ Carol A. RAYMOND ${ }^{2}$, and Christopher T. RUSSELL ${ }^{5}$ \\ ${ }^{1}$ Department of Geosciences, Virginia Tech, Blacksburg, Virginia 24061, USA \\ ${ }^{2}$ Jet Propulsion Laboratory, California Institute of Technology, Pasadena, California 91109, USA \\ ${ }^{3}$ Institut de Recherche d'Astrophysique et Planétologie, University of Toulouse, Toulouse, France \\ ${ }^{4}$ US Geological Survey, Astrogeology Science Center, Flagstaff, Arizona 86001, USA \\ ${ }^{5}$ Earth, Planetary, and Space Sciences, University of California, Los Angeles, California 90095, USA \\ *Corresponding author. E-mail: sdk@vt.edu
}

(Received 14 June 2017; revision accepted 30 January 2018)

\begin{abstract}
Thermal evolution modeling has yielded a variety of interior structures for Ceres, ranging from a modestly differentiated interior to more advanced evolution with a dry silicate core, a hydrated silicate mantle, and a volatile-rich crust. Here we compute the mass and hydrostatic flattening from more than one hundred billion three-layer density models for Ceres and describe the characteristics of the population of density structures that are consistent with the Dawn observations. We show that the mass and hydrostatic flattening constraints from Ceres indicate the presence of a high-density core with greater than a $1 \sigma$ probability, but provide little constraint on the density, allowing for core compositions that range from hydrous and/or anhydrous silicates to a mixture of metal and silicates. The crustal densities are consistent with surface observations of salts, water ice, carbonates, and ammoniated clays, which indicate hydrothermal alteration, partial fractionation, and the possible settling of heavy sulfide and metallic particles, which provide a potential process for increasing mass with depth.
\end{abstract}

\section{INTRODUCTION}

Ceres, the largest object in the asteroid belt at approximately $470 \mathrm{~km}$ in radius, is the most accessible volatile-rich proto-planetary body (McCord et al. 2011; Russell et al. 2016). Occupying a size intermediate between asteroids and planets, it is unclear to what extent differentiation processes, including the possible formation of a dense core, played a role in the evolution of Ceres. Ceres has a bulk density of $2162 \pm 8 \mathrm{~kg} \mathrm{~m}^{-3}$ (Park et al. 2016), which translates to a bulk composition of $\sim 17-27 \mathrm{wt} \%$ water ice (McCord et al. 2011) depending on the amount of water trapped in minerals. Thermal evolution modeling shows that radiogenic heating would be sufficient to melt the ice, fractionating the ice and rock components, and leading to the production of salts and hydrated silicates (McCord and Sotin 2005; see also McSween et al. 2018). Assuming a carbonaceous chondrite composition, thermal modeling prior to the arrival of Dawn predicted that Ceres would undergo some degree of thermal metamorphism at depth (McCord and Sotin 2005; Castillo-Rogez and McCord 2010; McCord and CastilloRogez 2018), accompanied by the migration of salt species toward the surface (Castillo-Rogez 2011). Thermal history calculations showed that the degree of heating, therefore the degree of alteration and differentiation, is dependent on both the long- and shortlived radiogenic element concentrations (see discussion in McCord and Castillo-Rogez 2018).

In spite of the absence of potential meteorite analogs (McSween et al. 2018), there is information regarding the composition of Ceres. Prior to Dawn, remote sensing with terrestrial and space-based telescopes indicated the possible presence of ammonium-bearing minerals (King et al. 1992) and brucite mixed with carbonate (Milliken and Rivkin 2009). Dawn's Visible and Infrared Spectrometer (VIR) has confirmed the presence of ammonia-bearing hydrated minerals (De Sanctis et al. 2016), as well as 
water ice, various types of carbonates, salts, serpentines, and organic material on Ceres' surface (De Sanctis et al. 2015, 2016, 2017; Ammannito et al. 2016), requiring extensive aqueous alteration (e.g., Castillo-Rogez et al. Forthcoming). Aqueous alteration results in the separation of the material into a briny liquid and lessmobile solid residue, and iron would be largely concentrated in the solid phase. Because of Ceres's large size, separation of the brine and residue by gravitational settling would be expected with the less dense brine forming the water, carbonate, salt, and organic-rich crust and more metallic phases sinking to the core.

Crater depths (Bland 2013; Bland et al. 2016) and topographic power spectra (Ermakov et al. 2017; Fu et al. 2017) constrain the rheology of the outer 40$100 \mathrm{~km}$ shell of Ceres. This in turn places constraints on the allowable composition of the outer $40-100 \mathrm{~km}$ shell. Crater relaxation limits the ice content of the outer $40 \mathrm{~km}$ of Ceres to be no more than $35 \%$, with a stronger (more viscous) component or components making up the bulk of the crust (Bland et al. 2016; Fu et al. 2017). Ahuna Mons, the only volcanic construction identified on Ceres's surface, indicates that brines at depth existed $<200 \mathrm{My}$ ago in at least one location (Ruesch et al. 2016), raising the possibility that a briny liquid subsurface layer may still be present, if not globally at least in local regions, as also suggested by Fu et al. (2017).

Gravity and topography from the Dawn spacecraft indicate that Ceres is a partially differentiated body (Park et al. 2016). The gravity and topography constraints allow for a range of density structures. For example, assuming a partially differentiated body with a CM chondrite composition, Park et al. inferred a $70-190 \mathrm{~km}$ thick outer shell with a density of 1680 $1950 \mathrm{~kg} \mathrm{~m}^{-3}$ and an interior with a density of 2460 $2900 \mathrm{~kg} \mathrm{~m}^{-3}$. They did not explore the possibility of further refining the interior into an intermediate density "mantle" and a higher density "core" as the problem becomes underconstrained. Additional insights come from combining the information contained in the gravity and topography data as done in the Ermakov et al. (2017) admittance analysis and $\mathrm{Fu}$ et al. (2017) topography relaxation modeling. In the former study, the outer shell density is matched by a mixture of volatiles and denser materials including silicates and salts, consistent with both the surface observations from the Dawn VIR instrument (Ammannito et al. 2016; De Sanctis et al. 2016, 2017) and the aforementioned crater (Bland et al. 2016) and geophysical constraints (Ermakov et al. 2017; Fu et al. 2017). The latter studies indicate a low core density $\left(\sim 2400 \mathrm{~kg} \mathrm{~m}^{-3}\right)$ that is interpreted as evidence for a cool thermal evolution.
Whether Ceres's differentiation proceeded as far as to produce a partially dehydrated silicate core, or even small iron core, remains an open question. Analysis of Dawn's GRaND data is consistent with several weight percent iron depletion in the equatorial average concentration of elemental iron relative to cosmochemical iron abundance or an average of $\mathrm{CI} / \mathrm{CM}$ chondrites (Prettyman et al. 2017). The joint measurements of iron and hydrogen concentrations imply that Ceres underwent some degree of ice-rock fractionation, if the starting composition was similar to carbonaceous chondrites (Prettyman et al. 2017).

In this work we address this question of whether Ceres may have a high-density core using the Dawn shape data and hydrostatic flattening constraints. Although Park et al. (2016) and Ermakov et al. (2017) restricted their focus to two-layer models for the radial density structure of Ceres, we consider a suite of threelayer density models varying the densities and radii of the layers (shells). After computing a large ensemble of density models that are consistent with the mass and shape observations, we compare the results with several surface compositions and discuss how these assumed compositions would impact the interior density structure.

\section{METHOD}

The triaxial shape of Ceres has been determined using Dawn spacecraft images obtained during the High Altitude Mapping Orbit phase of the Dawn encounter with Ceres. The images cover Ceres's surface with a resolution of about $140 \mathrm{~m}$ (Russell et al. 2016). Stereophotogrammetric reconstruction of these images enabled the production of a three-dimensional shape model of Ceres (Gaskell et al. 2008; Preusker et al. 2015). The global shape is fit by an ellipsoid with principal axes $a=4.831 \times 10^{5} \mathrm{~m}$ and $b=4.810 \times 10^{5} \mathrm{~m}$ in the equatorial plane and $c=4.459 \times 10^{5} \mathrm{~m}$ along the spin axis. The uncertainty in radius at each point is $\pm 200 \mathrm{~m}$ (Park et al. 2016). Ceres's shape departs from hydrostatic equilibrium by almost $2000 \mathrm{~m}$ between the equatorial principal axes, or $0.4 \%$. The inferred topographic flattening, $f=(a-c) / a$, is assumed to be dominated by rotation; however, for a body in hydrostatic equilibrium, the equatorial principal axes are equal (i.e., $a=b$ ). In the absence of internal density structures, the degree 2 order 1 and degree 2 order 2 coefficients of the spherical harmonic expansion of the gravitational potential are zero for a rotating body in hydrostatic equilibrium. The degree 2 coefficients of the Ceres gravity model from the LAMO orbit (Konopliv et al. 2017) are given in Table 1. The ratio of the degree 2 order 2 component of the gravitational potential to 
Table 1. Degree 2 coefficients of the gravity model from the LAMO orbit (Konopliv et al. 2017).

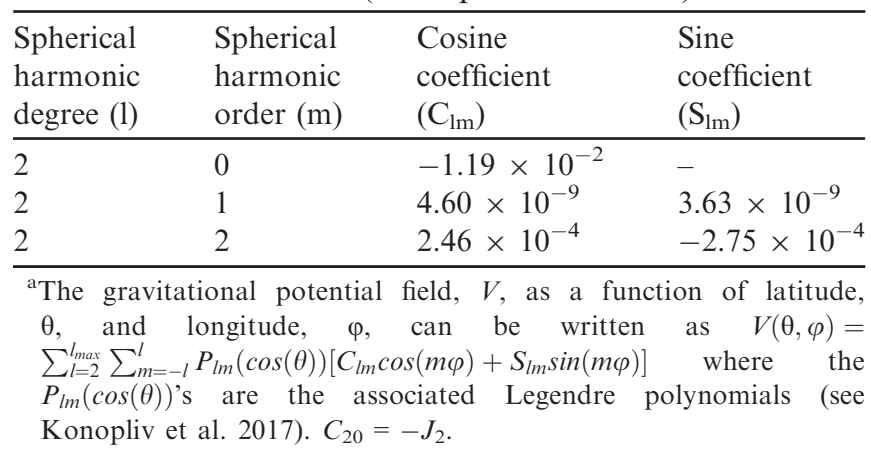

the degree 2 order 0 component of the gravitational potential is 0.031. Ermakov et al. (2017) assumed that the nonhydrostatic component of the degree 2 order 0 component of the gravitational potential is of the same order as the degree 2 order 2 component of the gravitational potential (i.e., $3 \%$ ). Because we found that a $\pm 3 \%$ range yielded too few successful models for statistical analysis, we expanded the uncertainty range of $J_{2}$ to $\pm 5 \%$ of the inferred value in the evaluation of density models. Tests using the topographic flattening, $f$, produced nearly identical results to those presented here.

Rambaux et al. (2015) showed that first-order hydrostatic equilibrium theory underestimates the difference between the equatorial and polar radii (i.e., $a-c)$ by as much as $1800 \mathrm{~m}$ for Ceres, which in turn leads to an underestimate of the extent of mass concentration. They found that a second-order expansion of Clairaut's equations is accurate to about $200 \mathrm{~m}$, consistent with uncertainty associated with the present Dawn shape and gravity models (Park et al. 2016). In this work, we solve Clairaut's equations to second order as described in Chambat et al. (2010).

The three-layer oblate spheroid density structure can be described by five parameters (three densities and the radii of the two density boundaries). Here for sake of familiarity, we adopt the terms "crust" for the outer shell, "mantle" for the intermediate shell, and "core" for the inner sphere. There are only two geophysical constraints used in this study, the effect of rotation on the gravitational potential, $J_{2}$, and the total mass of the satellite. We use a Monte Carlo approach, considering a more than 1,000,000 density surface, mantle, and core densities, and for each density triplet, we allow the core-mantle boundary and crust-mantle boundary radii to vary in $2 \mathrm{~km}$ increments (Table 2). The range of crust-mantle boundary radii is consistent with Ermakov et al. (2017). For the crust (outer shell), we randomly chose density values that range from 1150 to
Table 2. Parameter ranges for the three-shell density models.

\begin{tabular}{lc}
\hline Parameter & Evaluation range \\
\hline Outer shell (crustal) density $\left(\rho_{\mathrm{s}}\right)$ & $1150-1450 \mathrm{~kg} \mathrm{~m}^{-3}$ \\
Middle shell (mantle) density $\left(\rho_{\mathrm{m}}\right)$ & $1650-2450 \mathrm{~kg} \mathrm{~m}^{-3}$ \\
Inner sphere (core) density $\left(\rho_{\mathrm{c}}\right)$ & $1950-5150 \mathrm{~kg} \mathrm{~m}^{-3}$ \\
Core-mantle boundary radius $\left(r_{\mathrm{cmb}}\right)$ & $10-434 \mathrm{~km} \mathrm{in} 2 \mathrm{~km}$ \\
& steps \\
Crust-mantle boundary radius $\left(r_{\text {crust }}\right)$ & $10-434 \mathrm{~km}$ in $2 \mathrm{~km}$ \\
& steps \\
\hline
\end{tabular}

$1450 \mathrm{~kg} \mathrm{~m}^{-3}$, slightly expanding the range of crustal density models $\left(1200-1357 \mathrm{~kg} \mathrm{~m}^{-3}\right)$ that is consistent with admittance studies (Ermakov et al. 2017; Mitri et al. Forthcoming). For the mantle (middle shell), we randomly chose mantle densities ranging from 1650 to $2450 \mathrm{~kg} \mathrm{~m}^{-3}$, and for the core we randomly chose densities ranging from 1950 to $5150 \mathrm{~kg} \mathrm{~m}^{-3}$. The results are nearly identical to a fully grid-based approach where we considered the densities in 10 and $20 \mathrm{~kg} \mathrm{~m}^{-3}$ uniformly spaced increments spanning the ranges above (see also Table 2).

We reject density structures unless the resulting mass is within the uncertainty bounds of the observed mass (Park et al. 2016) (Table 3) and the normalized $J_{2}$ coefficient is within $5 \%$ of 0.0119 (Konopliv et al. 2017). Varying the misfit of the mass and $J_{2}$ constraints had only a minor impact on the distribution of density structures. The theory here assumes that each shell has a uniform density; therefore, the densities described here are best thought of as representing average densities within each of the three regions.

\section{RESULTS}

Using the approach described above, the 1,000,000 randomly selected density models resulted in the evaluation of more than a hundred billion density models. Of these, only 7078 or $0.000007078 \%$ were within observed mass and $J_{2}$ bounds. We plot histograms of the crustal density (Fig. 1a), mantle density (Fig. 1b), core density (Fig. 1c), core-mantle boundary radius (Fig. 1d), and crust-mantle boundary radius (Fig. 1e) in $5 \mathrm{~kg} \mathrm{~m}^{-3}$ density bins $\left(25 \mathrm{~kg} \mathrm{~m}^{-3}\right.$ for the core density) and $10 \mathrm{~km}$ radius bins. Of the five parameters, the mantle density histogram has a shape that more closely resembles a normal distribution with a mean of $2367 \mathrm{~kg} \mathrm{~m}^{-3}$ and a standard deviation of $26 \mathrm{~kg} \mathrm{~m}^{-3}$ (Fig. 1b). The crustal density histogram has no values below $1300 \mathrm{~kg} \mathrm{~m}^{-3}$ and the frequency of crustal densities increases with increasing density (Fig. 1a). The core density distribution peaks in the 
Table 3. Shape, mass, gravitational, and rotational parameters for Ceres.

\begin{tabular}{|c|c|}
\hline Parameter & Value \\
\hline Major radial axis $(a)$ & $483.1 \pm 0.2 \mathrm{~km}^{\mathrm{a}}$ \\
\hline Intermediate radial axis $(b)$ & $481.0 \pm 0.2 \mathrm{~km}^{\mathrm{a}}$ \\
\hline Minor radial axis $(c)$ & $445.9 \pm 0.2 \mathrm{~km}^{\mathrm{a}}$ \\
\hline Rotation rate $(\omega)$ & $1.9234 \times 10^{-4 b}$ \\
\hline Mass $(M)$ & $\begin{array}{l}938.416 \times 10^{18} \pm 0.013 \\
\times 10^{18} \mathrm{~kg}^{\mathrm{a}}\end{array}$ \\
\hline $\begin{array}{l}\text { Newton's gravitational } \\
\text { constant }(G)\end{array}$ & $6.67408 \times 10^{-11} \mathrm{~m}^{3} \mathrm{~kg}^{-1} \mathrm{~s}^{-2}$ \\
\hline Average density & $2162 \pm 8 \mathrm{~kg} \mathrm{~m}^{-3 a}$ \\
\hline $\begin{array}{l}\text { Normalized coefficient } \\
\text { of the reference } \\
\text { gravitational potential } \\
\text { ellipsoid }\left(J_{2}\right)\end{array}$ & $1.19 \times 10^{-2 \mathrm{c}}$ \\
\hline $\begin{array}{l}\text { Flattening } \\
\qquad(f=(c-a) / a)\end{array}$ & $7.4990 \times 10^{-2}$ \\
\hline $\begin{array}{l}\text { Centrifugal } \\
\text { /gravitational } \\
\text { potential ratio } \\
\left(m=\omega^{2} a^{3} / G M\right)\end{array}$ & $6.61648 \times 10^{-2}$ \\
\hline
\end{tabular}

2375-2425 $\mathrm{kg} \mathrm{m}^{-3}$ range with a long tail (Fig. 1c). The core-mantle boundary radii histogram (Fig. 1d) is nearly flat from 10 to $50 \mathrm{~km}$ with frequencies of $10 \%$ in each bin, and the frequencies drop almost linearly from 50 to $150 \mathrm{~km}$ with a long, low-frequency tail. The crust-mantle boundary radii distribution is a maximum of $50 \%$ at $35 \mathrm{~km}$ (the minimum value considered) and decreases to 0 at $425 \mathrm{~km}$ (Fig. 1d).

While the histograms in Fig. 1 indicate the frequency of occurrence of each of the five parameters, the parameters are not independent. To address this point, we create 2-D gridded distributions of density versus radius for the crust, mantle, and core. Considering the plot of density as a function of radius (Fig. 2a), for each density model, we create a function that counts the frequency of occurrence of the densityradii pairs by incrementing the value of the function the grid points corresponding to the crustal density (horizontal axis) for all radii (vertical axis) from the surface to the crust-mantle boundary. This produces a vertical line of grid points from the crust-mantle boundary to the surface at the appropriate crustal density. We repeat the same process for the mantle densities (from the core-mantle boundary to the crustmantle boundary, Fig. 2b) and for the core densities (from the center of the body to the core-mantle boundary, Fig. 2c). We then divide each value in the grid by the total number of acceptable models, giving a function between zero and one that shows the frequency
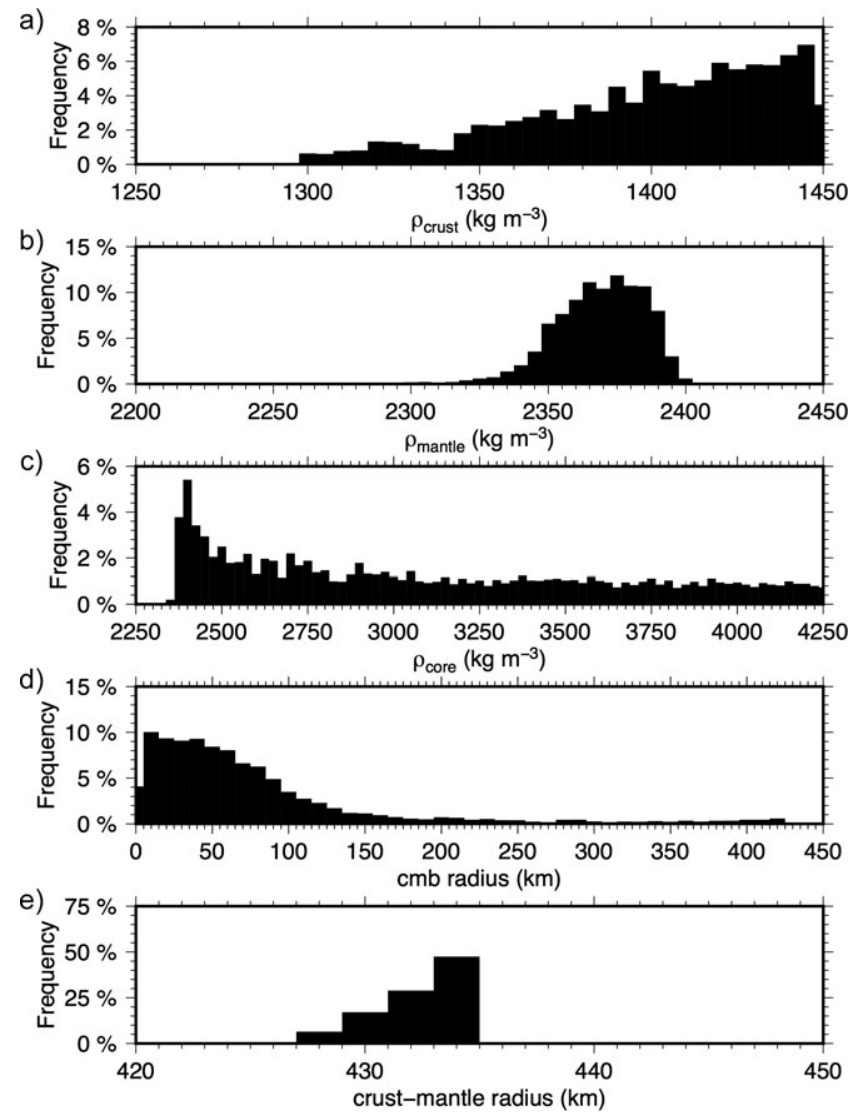

Fig. 1. Histograms of (a) crust, (b) mantle, and (c) core densities, as well as (d) core-mantle boundary radii, and (e) crust-mantle radii from the 7078 density models in the Monte Carlo simulation of hydrostatic flattening. Crust and mantle densities are binned in $10 \mathrm{~kg} \mathrm{~m}^{-3}$ increments, while core densities are binned in $25 \mathrm{~kg} \mathrm{~m}^{-3}$ increments. Crust-mantle boundary radii are binned in $2 \mathrm{~km}$ increments, while coremantle boundary radii are binned in $10 \mathrm{~km}$ increments.

of each density-radius pair. The vertical lines indicate the frequency of the density/thickness of the crust, mantle, and core, illustrating the trade-offs between the thickness and density of each layer. Considering the crust, the thickness of the crust increases as the density increases (Fig. 2a) and as was observed in the histogram plots, the higher crustal density models occur more frequently. For the mantle (Fig. 2b), once again, the mean density of $2367 \mathrm{~kg} \mathrm{~m}^{-3}$ stands out; however, we now see that there is a $20 \%$ probability that models have mantle densities in the range $2360-2380 \mathrm{~kg} \mathrm{~m}^{-3}$ over the radius range from $434 \mathrm{~km}$ to $80 \mathrm{~km}$ radius (tracing the 0.2 or $20 \%$ contour), while there is a 0.1 or $10 \%$ probability that the models have mantle densities in the range $2340-2385 \mathrm{~kg} \mathrm{~m}^{-3}$ over the radius range from $434 \mathrm{~km}$ to $25 \mathrm{~km}$ radius. For the core (Fig. 2c), the frequency plot has a number of vertical streaks (light gray), indicating that the number of successful is 
insufficient to create a smooth frequency map. However, the trend is clear, the core radius decreases as the core density increases. The most frequent core densities are the range $2385-2405 \mathrm{~kg} \mathrm{~m}^{-3}$. While this is larger than the mean mantle density $\left(2367 \mathrm{~kg} \mathrm{~m}^{-3}\right)$, indicating the likely presence of a core, it does partially overlap the $1 \sigma$ standard deviation of the mean mantle density $\left(2367+26=2393 \mathrm{~kg} \mathrm{~m}^{-3}\right)$.

Based on admittance modeling, Ermakov et al. (2017) found the best-fitting, two-layer density structures have crustal densities between 1200 and $1357 \mathrm{~kg} \mathrm{~m}^{-3}$ depending on the isostatic mechanism assumed in their analysis, with a preferred crustal density of $1287_{-87}^{+70} \mathrm{~kg} \mathrm{~m}^{-3}$ and a crustal thickness of $41_{-4.7}^{+3.2} \mathrm{~km}$. They estimate the mean interior density to be $2434_{-8}^{+5} \mathrm{~kg} \mathrm{~m}^{-3}$. In another admittance study, Mitri et al. (Forthcoming) report a crustal density of $1183_{-177}^{+141} \mathrm{~kg} \mathrm{~m}^{-3}$ and a crustal thickness of $38_{-6}^{+7} \mathrm{~km}$ with a mean interior density of $2442_{-94}^{+119} \mathrm{~kg} \mathrm{~m}^{-3}$. Both the Ermakov et al. and the Mitri et al. results overlap when taking into account the range of reported uncertainties. The three-layer models described above have an average mantle density value of $2367 \mathrm{~kg} \mathrm{~m}^{-3}$, smaller than and outside of the uncertainty range of the interior densities reported in the Ermakov et al. study even when taking into account the $1-\sigma$ standard deviation (2341-2393 $\mathrm{kg} \mathrm{m}^{-3}$ ), although it does overlap the more generous bounds of the Mitri et al. study. The difference in mean mantle density is not surprising because the crustal densities in the hydrostatic density models are always greater than $1300 \mathrm{~kg} \mathrm{~m}^{-3}$, which is larger than the preferred value from the Ermakov et al. study. It is not surprising that the admittance and hydrostatic studies find slightly different density structures because the degree 2 and higher order degree admittance results do not agree (see fig. 11 in Ermakov et al. 2017). There is something unusual about Ceres at degree 2.

Figure 3 shows the resulting gridded frequency maps after truncating the population of acceptable density models from this study to the range of crustal densities inferred from the Ermakov et al. study (1200$1357 \mathrm{~kg} \mathrm{~m}^{-3}$ ). In this truncated distribution, the mean mantle density rises slightly to $2379 \mathrm{~kg} \mathrm{~m}^{-3}$ and the standard deviation is reduced to $10 \mathrm{~kg} \mathrm{~m}^{-3}$. The most frequent core densities decrease to $2385-2395 \mathrm{~kg} \mathrm{~m}^{-3}$ for a core that can be as much as $350 \mathrm{~km}$ in radius, with additional peaks in the density ranges 2510 $2570 \mathrm{~kg} \mathrm{~m}^{-3}(<80 \mathrm{~km}$ in radius $), 2600-2620 \mathrm{~kg} \mathrm{~m}^{-3}$ $\left(<30 \mathrm{~km}\right.$ in radius), $2645-2665 \mathrm{~kg} \mathrm{~m}^{-3}(<30 \mathrm{~km}$ in radius), and $2685-2705 \mathrm{~kg} \mathrm{~m}^{-3}(<30 \mathrm{~km}$ in radius $)$.

While instructive, these figures still do not illustrate the trade-offs among the five parameters. For example, if the density of the crust increases, then in order to a)

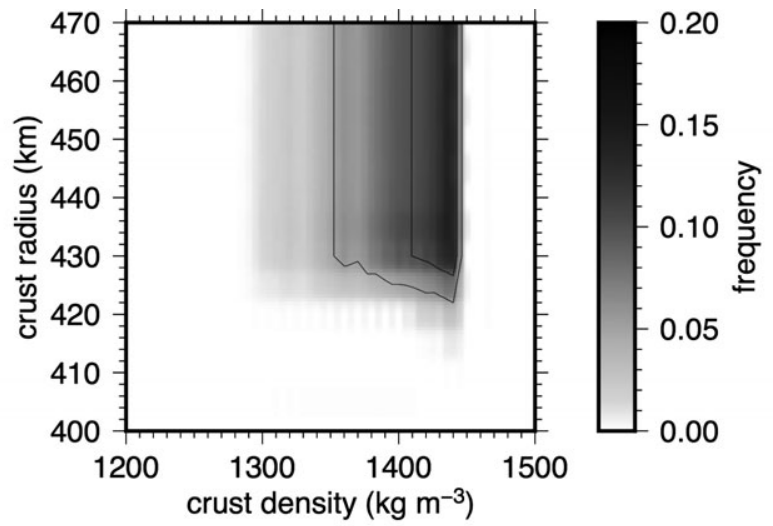

b)

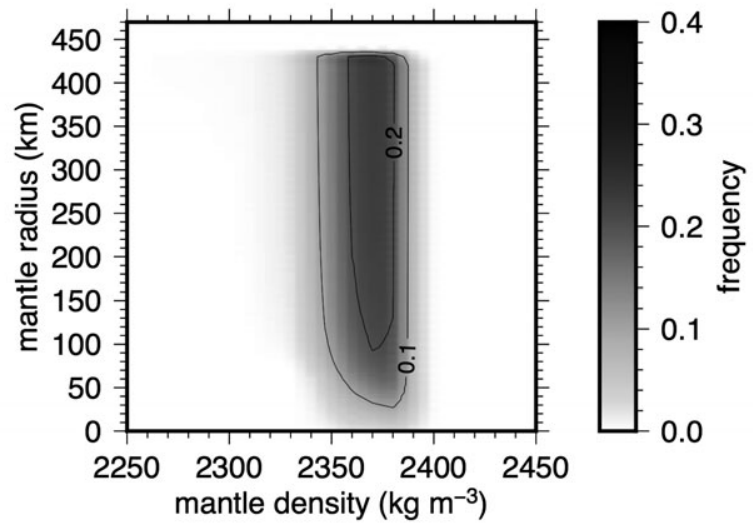

c)

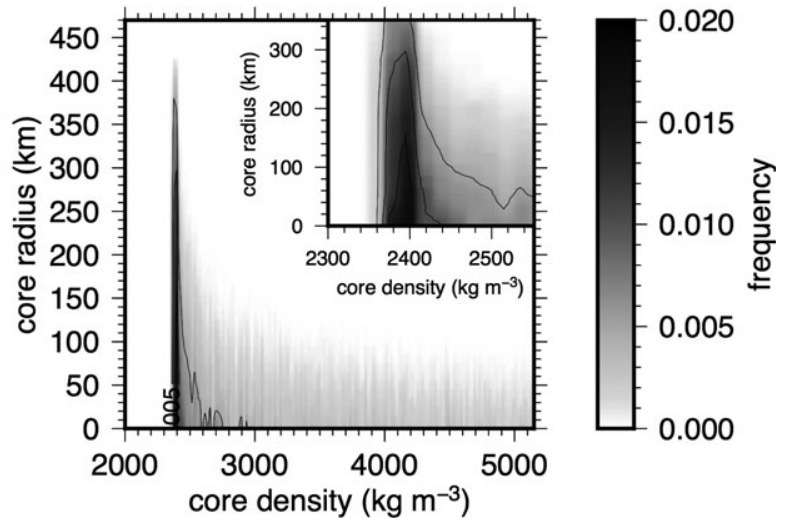

Fig. 2. Plots of frequency of (a) crust, (b) mantle, and (c) core densities and radii (thickness) for the population of models from the Monte Carlo simulation. The vertical streaks are the result of the assumed uniform density within each shell.

maintain the mass constraint, either the density of one of the other layers must decrease or the thickness of a dense shell must be reduced. The most direct way to address the possible presence of a core is to plot a histogram of the density difference between the core and mantle for each model in $50 \mathrm{~kg} \mathrm{~m}^{-3}$ bins. The results for the full population (Fig. 4a) and the populations truncated to the Ermakov et al. crustal density range (Fig. $4 \mathrm{~b}$ ) have similar trends with $2.5 \%$ of the models having equal mantle and core densities and 
a)
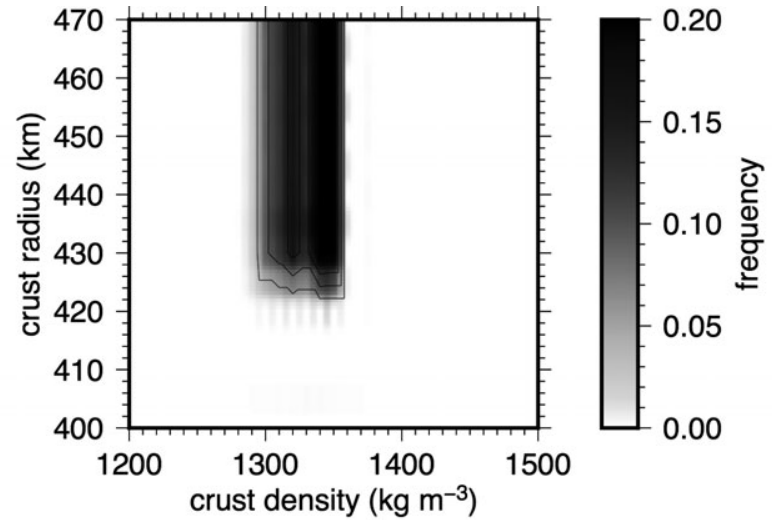

b)

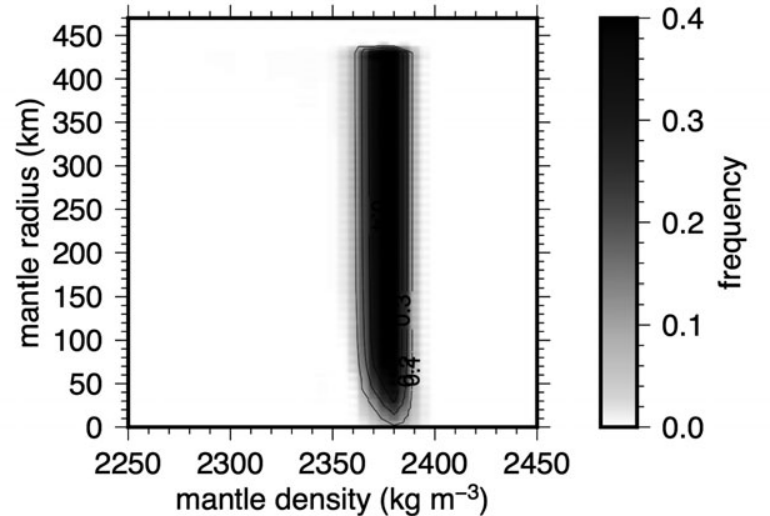

c)

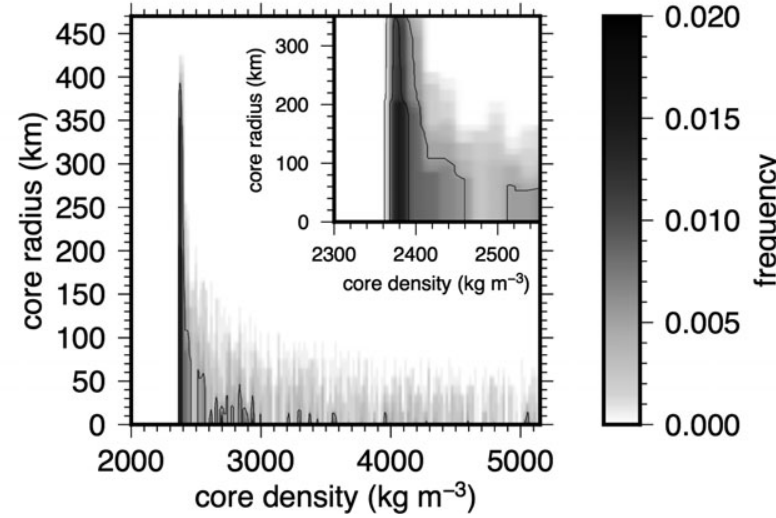

Fig. 3. Plots of frequency of (a) crust, (b) mantle, and (c) core densities and radii (thickness) for the population of models restricted to the preferred crustal density range of Ermakov et al. (2017). The vertical streaks are the result of the assumed uniform density within each shell.

a peak density difference in the $50-200 \mathrm{~kg} \mathrm{~m}^{-3}$ range with long, noisy tails in the $1-2 \%$ frequency range. While the trend in histogram in Fig. $4 \mathrm{~b}$ matches that in Fig. 4a, the distribution is not as smooth, likely because there are fewer models in the distribution.

While the frequency in each individual bin in the histogram is small, the probability that the density is within the range between $\mathrm{A}$ and $\mathrm{B}$ is the integral of the probability distribution function between $\mathrm{A}$ and $\mathrm{B}$. As a a)

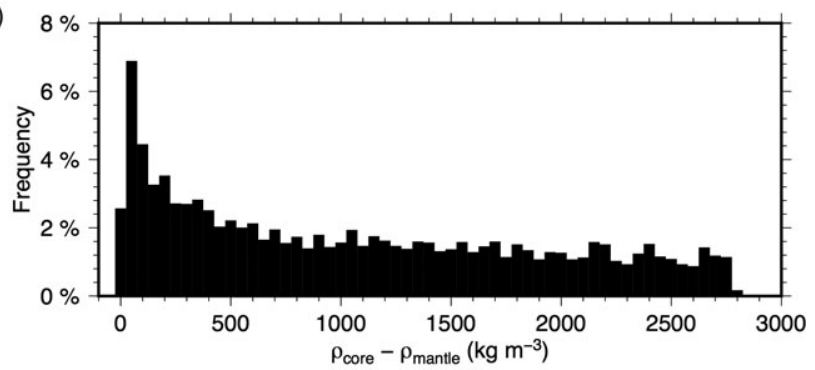

b)

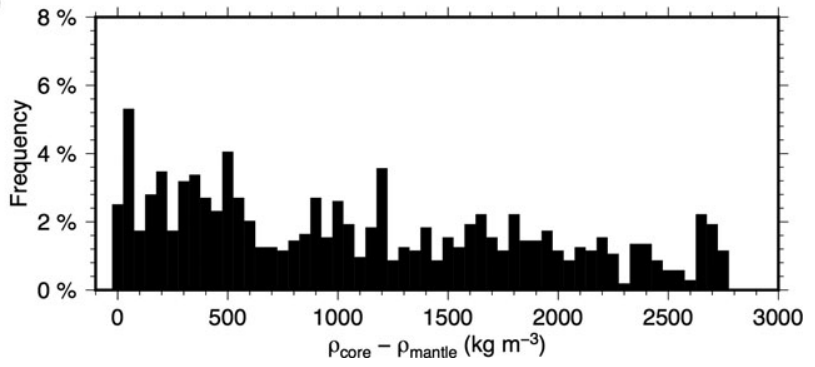

Fig. 4. Histograms of the density difference between the mantle and core from the Monte Carlo model population for (a) the entire population and (b) the population restricted to the preferred crustal density range of Ermakov et al. (2017) both in $50 \mathrm{~kg} \mathrm{~m}^{-3}$ bins.

histogram is a discrete approximation of a probability distribution function, we can approximate the integral by plotting the cumulative probability histogram (Fig. 5) for the two populations described in Fig. 4. While the curves differ in minor details, in each case, more than $67 \%$ of the models $(1 \sigma)$ have core densities at least $300 \mathrm{~kg} \mathrm{~m}^{-3}$ greater than the corresponding mantle density.

\section{DISCUSSION}

It is not surprising that the mantle density in the three-layer models is smaller than the mantle density in the two-layer studies, because including a dense core necessitates reducing either the crust or mantle density. However, the mean density of the mantle plus core, appropriately weighted by the respective volumes of the mantle and core from this study is still slightly smaller than the mean interior density reported in the two-layer studies. The hydrostatic theory results here consistently produce higher density, thinner crustal layers than the admittance results. The difference is currently not understood and could be related to lateral variations in crustal structure that invalidate the assumption of isostasy in the admittance studies, despinning of Ceres (Mao and McKinnon 2016), or some other as yet unknown processes. As we restrict the crustal densities to the preferred range reported by Ermakov et al., we still find a lower mantle density than reported by 

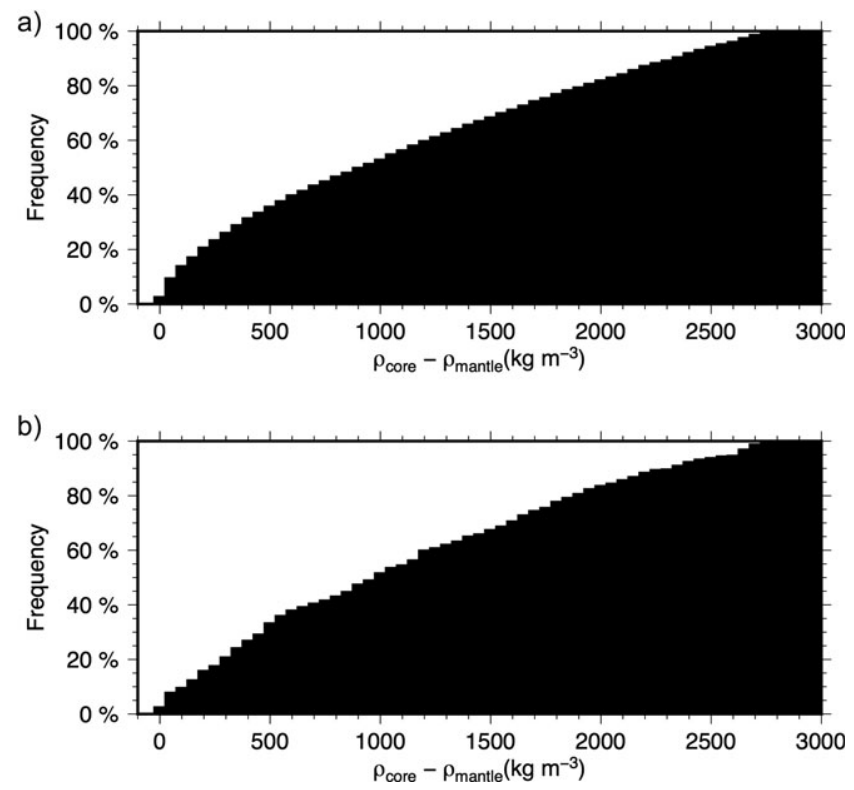

Fig. 5. Cumulative histograms of the density difference between the mantle and core from the Monte Carlo model population for (a) the entire population and (b) the population restricted to the preferred crustal density range of Ermakov et al. (2017) both in $50 \mathrm{~kg} \mathrm{~m}^{-3}$ bins.

Ermakov et al. This is likely because in two-layer modeling, the mass concentration with depth can only be accommodated by the position of the crust/mantle boundary and the density increase across that boundary. In three-layer modeling, the mass concentration can be accommodated by two density boundaries and two corresponding density increases, thus the first density increase in the three-layer model is almost certainly going to be smaller than in the corresponding two-layer case.

Unfortunately, we are not able to place constraints on the core density beyond stating that the denser the core, the smaller it must be. A core with a composition ranging from serpentine to iron (e.g., McCord and Sotin 2005) has been proposed for Ceres based on thermal history modeling. Taking the mantle density of $2367 \mathrm{~kg} \mathrm{~m}^{-3}$, core-mantle density differences up to $500 \mathrm{~kg} \mathrm{~m}^{-3}$ would be consistent with hydrated silicates, and this core density range includes close to $30 \%$ of the models. An anhydrous silicate core spans a core-mantle density difference range of $500-1500 \mathrm{~kg} \mathrm{~m}^{-3}$ and approximately $30 \%$ of the models have core densities from 2700 to $3700 \mathrm{~kg} \mathrm{~m}^{-3}$. Finally, core densities greater than $3700 \mathrm{~kg} \mathrm{~m}^{-3}$ require a dense component in addition to silicates, possibly indicating an increase in Fe content and $40 \%$ of the models have core densities greater than $3700 \mathrm{~kg} \mathrm{~m}^{-3}$.

The inferred crustal density range of 1200$1357 \mathrm{~kg} \mathrm{~m}^{-3}$ is consistent with low-density mineral phases including water, carbonates, salts, serpentine, ammonia-bearing hydrated minerals, and organic material (Ammannito et al. 2016; De Sanctis et al. 2016, 2017). The crustal composition of water ice, carbonates, phyllosilicates, and salt and/or clathrate hydrate phases is most consistent with models of an ancient ocean layer that underwent progressive freezing, leading to the concentration of salts (Neveu and Desch 2015; Fu et al. 2017). It is possible that rocky particles enriched in magnetite and sulfides were concentrated at depth, per their greater densities, during the differentiation phase, when Ceres held a deep ocean. A similar model was proposed for icy satellites by Scott et al. (2002). Using data from Dawn's Gamma Ray and Neutron Detector (GRaND), Prettyman et al. (2017) determined the equatorial average concentration of elemental iron at the surface of Ceres is $16 \pm 1 \mathrm{wt} \%$. This is $\sim 10 \%$ lower than cosmochemical iron abundances (e.g., McDonough and Sun 1995) and 15-30\% lower than CM and CI chondrite averages (Lodders and Fegley 1998), and may provide a possible mechanism and source for the highdensity component in the large core density models.

\section{CONCLUSIONS}

Solving Clairaut's equation to second order for $J_{2}$, the gravitational potential due to rotation, we conclude that the Dawn shape and gravity models indicate the presence of a core within Ceres with a probability greater than $1 \sigma$. There is little constraint on the density of the core with acceptable core densities, allowing for compositions of hydrous and/or anhydrous silicates as well as a mixture of a denser component with the silicates. Throughout the interior of Ceres, excluding approximately the innermost $100 \mathrm{~km}$ radius, the average density is $2367 \pm 26 \mathrm{~kg} \mathrm{~m}^{-3}$, slightly smaller than the average interior density from admittance modeling studies (Ermakov et al. 2017; Mitri et al. Forthcoming). The presence of a dense core is consistent with laboratory simulations of heavy mineral (e.g., magnetite, sulfide-rich particles) separation under hydrothermal conditions and preferential concentration at depth when Ceres held an ocean. Denser material may also point to the presence of anhydrous silicates, either remnants from the originally accreted material or resulting from partial dehydration.

Acknowledgments - We thank Walter Kiefer, Anton Ermakov, and an anonymous reviewer for their constructive comments. We thank Frédéric Chambat for making the Matlab program based on Chambat et al. (2010) available. S. D. K. is supported by NASA award NNX15AI30G from the Dawn at Ceres Guest Investigator Program. We thank the Dawn team for the development, cruise, orbital insertion, and operations of the Dawn spacecraft at Ceres. 
C. T. R. is supported by the Discovery Program through contract NNM05AA86C to the University of California, Los Angeles.

A portion of this work was performed at the Jet Propulsion Laboratory, California Institute of Technology, under contract with NASA. The authors have no conflict of interest to declare.

\section{Editorial Handling-Dr. Hap McSween}

\section{REFERENCES}

Ammannito E., De Sanctis M. C., Ciarniello M., Frigeri A., Carrozzo F. G., Combe J.-P., Ehlmann B. L., Marchi S., McSween H. Y., Raponi A., Toplis M. J., Tosi F., Castillo-Rogez J. C., Capaccioni F., Capria M. T., Fonte S., Giardino M., Jaumann R., Longobardo A., Joy S. P., Magni G., McCord T. B., McFadden L. A., Palomba E., Pieters C. M., Polanskey C. A., Rayman M. D., Raymond C. A., Schenk P., Zambon F., and Russell C. T. 2016. Distribution of phyllosilicates on Ceres. Science 353: aaf4279.

Bland M. T. 2013. Predicted crater morphologies on Ceres: Probing internal structure and evolution. Icarus 226:510521.

Bland M. T., Raymond C. A., Schenk P. M., Fu R. R., Park R., Kneissl T., Hiesinger H., Marchi S., King S. D., Castillo-Rogez J. C., and Russell C. T. 2016. Composition and structure of the shallow subsurface of Ceres revealed by crater morphology. Nature Geoscience 9:538-543.

Castillo-Rogez J. C. 2011. Ceres-Neither a porous nor salty ball. Icarus 215:599-602.

Castillo-Rogez J. C. and McCord T. B. 2010. Ceres' evolution and present state constrained by shape data. Icarus 205:443-459.

Castillo-Rogez J. C., Neveu M., McSween H. Y., Fu R., and Toplis M. Forthcoming. Insights into Ceres' evolution from surface composition. Meteoritics \& Planetary Science.

Chambat F., Ricard Y., and Valette B. 2010. Flattening of the Earth: Further from hydrostaticity than previously estimated. Geophysical Journal International 183:727-732.

Chamberlain M. A., Sykes M. V., and Esquerdo G. A. 2007. Ceres lightcurve analysis-Period determination. Icarus 188:451-456.

De Sanctis M. C., Ammannito E., Raponi A., Marchi S., McCord T. B., McSween H. Y., Capaccioni F., Capria M. T., Carrozzo F. G., Ciarniello M., Longobardo A., Tosi F., Fonte S., Formisano M., Frigeri A., Giardino M., Magni G., Palomba E., Turrini D., Zambon F., Combe J.P., Feldman W., Jaumann R., McFadden L. A., Pieters C. M., Prettyman T., Toplis M., Raymond C. A., and Russell C. T. 2015. Ammoniated-phyllosilicates on dwarf planet Ceres with a likely outer solar system origin. Nature 528:241-244.

De Sanctis M. C., Raponi A., Ammannito E., Ciarniello M., Toplis M. J., McSween H. Y., Castillo-Rogez J. C., Ehlmann B. L., Carrozzo F. G., Marchi S., Tosi F., Zambon F., Capaccioni F., Capria M. T., Fonte S., Formisano M., Frigeri A., Giardino M., Longobardo A., Magni G., Palomba E., McFadden L. A., Pieters C. M., Jaumann R., Schenk P., Mugnuolo R., Raymond C. A., and Russell C. T. 2016. Bright carbonate deposits as evidence of aqueous alteration on Ceres. Nature 536:5457.

De Sanctis M. C., Ammannito E., McSween H. Y., Raponi A., Marchi S., Capaccioni F., Capria M. T., Carrozzo G., Cioarneillo M., Fonte S., Formisano M., Frigeri A., Giardino M., Longobardo A., Magni G., McFadden L. A., Palomba E., Pieters C. M., Tosi F., Zambon F., Raymond C. A., and Russell C. T. 2017. Localized aliphatic organic material on the surface of Ceres. Nature 355:719-722.

Ermakov A. I., Fu R. R., Castillo-Rogez J. C., Raymond C. A., Park R. S., Preusker F., Russell C. T., Smith D. E., and Zuber M. T. 2017. Constraints on Ceres' internal structure and evolution from its shape and gravity measured by the Dawn spacecraft. Journal of Geophysical Research 122:2267-2293.

Fu R. R., Ermakov A. I., Marchi S., Castillo-Rogez J. C., Raymond C. A., Hager B. H., Zuber M. T., King S. D., Bland M. T., De Sanctis M. C., Preusker F., Park R. S., and Russell C. T. 2017. The interior structure of Ceres as revealed by surface topography. Earth and Planetary Science Letters 476:153-164.

Gaskell R. W., Barnouin-Jha O. S., Scheeres D. J., Konopliv A. S., Mukai T., Abe S., Saito J., Ishiguro M., Kubota T., Hashimoto T., Kawaguchi J., Yoshikawa M., Shirakawa K., Kominato T., Hirata N., and Demura H. 2008. Characterizing and navigating small bodies with imaging data. Meteoritics \& Planetary Science 43:10491061 .

King T. V., Clark R. N., Calvin W. M., Sherman D. M., and Brown R. H. 1992. Evidence for ammonium-bearing minerals on Ceres. Science 255:1551-1553.

Konopliv A. S., Park R. S., Vaughan A. T., Bills B. G., Asmar S. W., Ermakov A. I., Rambaux N., Raymond C. A., Castillo-Rogez J. C., Russell C. T., Smith D. E., and Zuber M. T. 2017. The Ceres gravity field, spin pole, rotation period and orbit from the Dawn radio-metric tracking and optical data. Icarus 299:411-429.

Lodders K. and Fegley B. Jr. 1998. The planetary scientist's companion. Oxford University Press on Demand.

Mao X. and McKinnon W. B. 2016. Internal structures of Ceres and Enceladus: Comparisons and contrasts (abstract \#1637). 47th Lunar and Planetary Science Conference. CD-ROM.

McCord T. B. and Castillo-Rogez J. C. Forthcoming. Ceres evolution, before and after Dawn. Meteoritics \& Planetary Science.

McCord T. B. and Sotin C. 2005. Ceres: Evolution and current state. Journal of Geophysical Research 110 (EO5009):1-14.

McCord T. B., Castillo-Rogez J. C., and Rivkin A. 2011. Ceres: Its origin, evolution and structure and Dawn's potential contribution. Space Science Reviews 163:63-76.

McDonough W. F. and Sun S. S. 1995. The composition of the Earth. Chemical Geology 120:223-253.

McSween H. Y., Emery J. P., Rivkin A. S., Toplis M. J., Castillo-Rogez J. C., Prettyman T. H., DeSanctis M. C., Pieters C. M., Raymond C. A., and Russell C. T. 2018. Carbonaceous chondrites as analogs for the composition and alteration of Ceres. Meteoritics \& Planetary Science. https://doi.org/10.1111/maps.12947

Milliken R. E. and Rivkin A. S. 2009. Brucite and carbonate assemblages from altered olivine-rich materials on Ceres. Nature Geoscience 2:258. 
Mitri G., Park R. S., Rambaux N., Castillo-Rogez J. C., Russell C. T., and Raymond C. A. Forthcoming. Crustal structure and internal differentiation of the dwarf planet Ceres. Journal of Geophysical Research.

Neveu M. and Desch S. J. 2015. Geochemistry, thermal evolution, and cryovolcanism on Ceres with a muddy ice mantle. Geophysical Research Letters 42:10,197-10,206.

Park R. S., Konopliv A. S., Bills B. G., Rambaux N., Castillo-Rogez J. C., Raymond C. A., Vaughan A. T., Ermakov A. I., Zuber M. T., Fu R. R., Toplis M. J., and Russell C. T. 2016. A partially differentiated interior for (1) Ceres deduced from its gravity field and shape. Nature 537:515-517.

Prettyman T. H., Yamashita N., Toplis M. J., McSween H. Y., Schorghofer N., Marchi S., Feldman W. C., Castillo-Rogez J., Forni O., Lawrence D. J., Ammannito E., Ehlmann B. L., Sizemore H. G., Joy S. P., Polanskey C. A., Rayman M. D., Raymond C. A., and Russell C. T. 2017. Extensive water ice within Ceres' aqueously altered regolith: Evidence from nuclear spectroscopy. Science 355:55-59.

Preusker F., Scholten F., Matz K.-D., Roatsch T., Willner K., Hviid S. F., Knollenberg J., Jorda L., Gutiérrez P. J., Kührt E., Mottola S., A'Hearn M. F., Thomas N., Sierks H., Barbieri C., Lamy P., Rodrigo R., Koschny D., Rickman H., Keller H. U., Agarwal J., Barucci M. A., Bertaux J.-L., Bertini I., Cremonese G., Da Deppo V., Davidsson B., Debei S., De Cecco M., Fornasier S., Fulle M., Groussin O., Güttler C., Ip W.-H., Kramm J. R., Küppers M., Lara L. M., Lazzarin M., Lopez Moreno J. J., Marzari F., Michalik H., Naletto G., Oklay N., Tubiana C., and Vincent J.-B. 2015. Shape model, reference system definition, and cartographic mapping standards for comet $67 \mathrm{P} /$ Churyumov-Gerasimenkostereo-photogrammetric analysis of Rosetta/OSIRIS image data. Astronomy \& Astrophysics 583:A33.

Rambaux N., Chambat F., and Castillo-Rogez J. C. 2015. Third-order development of shape, gravity, and moment of inertia for highly flattened celestial bodies. Application to Ceres, $A \& A$ 584:A127.

Ruesch O., Platz T., Schenk P., McFadden L. A., CastilloRogez J. C., Quick L. C., Byrne S., Preusker F., O'Brien D. P., Schmedemann N., Williams D. A., Li J.-Y., Bland M. T., Hiesinger H., Kneissl T., Neesemann A., Schaefer M., Pasckert J. H., Schmidt B. E., Buczkowski D. L., Sykes M. V., Nathues A., Roatsch T., Hoffmann M., Raymond C. A., and Russell C. T. 2016. Geologically recent cryo-volcanic activity on Ceres. Science 353:6303.

Russell C. T., Raymond C. A., Ammannito E., Buczkowski D. L., De Sanctis M. C., Hiesinger H., Jaumann R., McSween H. Y., Nathues A., Park R. S., Pieters C. M., Prettyman T. H., McCord T. B., McFadden L., Mottola S., Zuber M. T., Joy S. P., Polanskey C., Rayman M. D., Castillo-Rogez J. C., Combe J. P., Ermakov A. I., Fu R. R., Hoffmann M., Jia Y. D., Li J.-Y., Marchi S., Preusker F., Roatsch T., Ruesch O., Schenk P., Villarreal M. N., and Yamashita N. 2016. Dawn arrives at Ceres: Exploration of a volatile-rich dwarf planet. Science 353:1008-1010.

Scott H. P., Wililams Q., and Ryerson F. J. 2002. Experimental constraints on the chemical evolution of large icy satellites. Earth and Planetary Science Letters 203:399-412. 